\section{A Mouthguard for Endoscopic Examination of Patients with Oral Submucous Fibrosis and Ankylosis of the Temporomandibular Joint}

A mouthguard is invariably used during upper gastrointestinal endoscopy. While the conventional mouthguards provided with Olympus adult endoscopes (XQ-20 or $30)$ or pediatric endoscopes (PQ-20 or 30) are useful in the majority of cases, they cannot be used in patients with ankylosis of the temporomandibular joint or patients with severe oral submucous fibrosis (1) when the patient is not able to open his or her mouth sufficiently to accommodate the mouthguard. A simple, cheap and disposable mouthguard has been devised for use in such situations.

The plunger of a $5 \mathrm{ml}$ disposable plastic syringe was removed, and the distal $2 \mathrm{~cm}$ of the barrel (towards the barrel guard) was cut off and used as a disposable mouthguard. The outer diameter of this improvised mouthguard is $14 \mathrm{~mm}$, as compared to $20 \mathrm{~mm}$ in the one supplied with the pediatric endoscope (Figure 1). A pediatric endoscope (Olympus PQ-20 or 30 ) with an outer diameter of $9 \mathrm{~mm}$ was easily passed through the mouthguard, and upper gastrointestinal endoscopy was performed without any difficulty. The mouthguard can even be compressed slightly and used in patients with severe oral submucous fibrosis or ankylosis of the temporomandibular joint. The minimum diameter of the mouthguard after compression is only $12 \mathrm{~mm}$. This mouthguard has been successfully used in eleven patients with submucous fibrosis and three patients with ankylosis of the temporomandibular joint when a conventional mouthguard could not be used. There were no complications due to the mouthguard.

\section{S. P. Misra, M. Dwivedi}

Dept. of Gastroenterology, M. L. N. Medical College, Allahabad, India

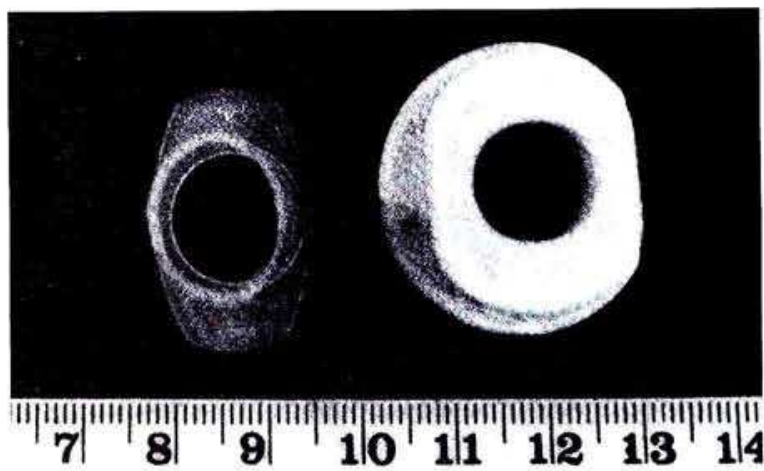

Figure 1: The diameter of the improvised mouthguard is considerably less than that provided with a pediatric upper gastrointestinal endoscope

\section{Reference}

1. Jayanthi V, Probert CSJ, Sher KS, Mayberry JF. Oral submucosal fibrosis: a preventable disease. Gut 1992; 33: 4-6.
Corresponding Author

S. P. Misra, M.D.

Dept. of Gastroenterology

M. L. N. Medical College

Allahabad 211001 , India

Fax: +91-532-61 1420

E-mail: mravi@wiprobt.ems.vsnl.net.in 